\title{
A Computational Study of Lean Ammonia Premixed Flame in MILD Combustion Conditions
}

\author{
Ruslan Khamedov*,Wonsik Song ${ }^{\dagger}$, Francisco E. Hernández Pérez ${ }^{\ddagger}$, and Hong G. $\operatorname{Im}^{\S}$ \\ Clean Combustion Research Center, King Abdullah University of Science and Technology (KAUST), \\ Thuwal 23955-6900, Saudi Arabia
}

\begin{abstract}
To provide fundamental insights into the heat release and emission characteristics of ammonia flames in moderate or intense low-oxygen dilution (MILD) conditions, numerical simulations in one-dimensional laminar and two-dimensional turbulent flame configurations are conducted. The analysis shows that for the ammonia MILD flame, lower $\mathrm{NO}_{\mathrm{x}}$ emissions can be achieved while improving flame stability characteristics. In addition, the propagation of ammonia flames into forced turbulent flows in a channel is examined in terms of turbulent flame speed and surface area enhancement. The normalized flame speed and flame surface area are well correlated, and show qualitatively similar behavior for the MILD and non-MILD flames.
\end{abstract}

\section{Introduction}

A MMONIA has recently gained significant interest across scientific and industrial communities as a future fuel. The role of this combustible inorganic substance in the decarbonization of the energy sector is accompanied by the potential to pave the way towards a hydrogen society. Ammonia is comprised by almost $18 \%$ hydrogen in terms of mass and is accepted as one of the hydrogen-combustion enablers with mature and economically viable infrastructure for transportation and storage [1]. Unlike hydrogen, ammonia is relatively easily transported in liquid form in low-pressure vessels. Besides, the high octane number of ammonia (around 130) makes it feasible to use in internal combustion engines to eliminate knocking [2-4].

Despite its potential as alternative fuel, ammonia oxidation in pure form faces some challenges that need to be tackled before it is effectively applied in combustion systems. Laboratory-scale experiments have shown that ammonia combustion exhibits flame instabilities and high nitrogen oxide $\left(\mathrm{NO}_{\mathrm{x}}\right)$ emissions [1, 5, 6]. The $\mathrm{NO}_{\mathrm{x}}$ in ammonia flames are mainly produced through the fuel-NO pathway and are significantly high in lean conditions, which in turn requires the implementation of costly after-treatment systems to reduce emission to acceptable levels. Nonetheless, emissions can be minimized by burning ammonia in fuel-rich conditions, but leading to the necessity to eliminate unburned ammonia and $\mathrm{N}_{2} \mathrm{O}$, another greenhouse gas, from the exhaust gases [7, 8]. The issue associated with the slow flame speed of pure ammonia (around 6-8 $\mathrm{cm} / \mathrm{s}$ at a stoichiometric condition [9]) and high ignition energy [5] can be overcome by blending ammonia with other fuels to boost the reactivity of the mixture. Co-firing ammonia with hydrogen is a feasible way to reach carbon-free combustion and enhance combustion characteristics (ignitability and stability).

Another pragmatic approach of ammonia utilization in stationary power generation is applying the promising combustion concept known as moderate or intense low-oxygen dilution (MILD) combustion, which offers unique potential for simultaneously increasing combustion efficiency and reducing pollutant emissions [10, 11]. MILD combustion involves preheating and dilution. The highly preheated and diluted nature of the reactant mixture enhances flame stability and reduces thermal- $\mathrm{NO}_{\mathrm{x}}$ formation. Practically, the MILD combustion concept shares some common features with modern engine concepts such as the homogeneous charge compression ignition (HCCI) engine and the exhaust gas recirculation (EGR) process.

The physical insights of MILD combustion are difficult to unravel due to atypical transport-chemistry interaction, chemical kinetics, and dominant characteristic physical scales [12, 13]. Recently, Sorrentino et al. [14] studied the stability and emission characteristics of ammonia MILD flames for various equivalence ratios and inlet temperatures in a cyclonic burner. The lower $\mathrm{NO}_{\mathrm{x}}$ under the nearly stoichiometric condition and stable combustion at burner temperatures above $1300 \mathrm{~K}$ were experimentally found in this study. Moreover, Ariemma et al. [15] experimentally analyzed the

\footnotetext{
*Ph.D. Student, Clean Combustion Research Center, ruslan.khamedov@kaust.edu.sa.

$\dagger$ Ph.D. Student, Clean Combustion Research Center, wonsik.song@kaust.edu.sa.

${ }^{\ddagger}$ Research Scientist, Clean Combustion Research Center, francisco.hernandezperez.1@kaust.edu.sa, AIAA Member.

${ }^{\S}$ Professor, Clean Combustion Research Center, hong.im@kaust.edu.sa, AIAA Associate Fellow.
} 
effect of water addition to the unburned mixture, boosting $\mathrm{NO}_{\mathrm{x}}$ reduction by the $\mathrm{DeNO}_{\mathrm{x}}$ process in a cyclonic burner. The study concluded that water addition has a positive effect in terms of emissions. However, further fundamental understanding of the underlying physical and chemical processes of ammonia MILD flames is needed.

To this end, this research work is aimed at providing fundamental insights into ammonia premixed flame characteristics in MILD conditions through high-fidelity simulations. Phenomenological observations are discussed in terms of heat release characteristics, dominant reaction pathways, emissions distribution, and turbulence-flame interplay. Turbulent ammonia flames propagating into forced turbulent flows in a channel are further examined in terms of turbulent flame speed and curvature effect from global perspectives. In the following, the computational method and conditions are introduced, followed by the discussion about laminar and turbulent ammonia flame behavior. Lastly, concluding remarks are provided.

\section{Computational Methods and Conditions}

Fundamental combustion characteristics of ammonia flames at MILD conditions, such as the heat release rate and emissions, are first analyzed for the one-dimensional planar flame configuration. The simulations have been carried out with the Cantera library [16] using a chemical kinetic model by Otomo et al. [17] which consists of 32 species and 255 elementary reactions.

By definition the MILD combustion mode requires intense dilution and preheating of the reactant mixture. Additionally, it is required to keep the temperature of reactants $\left(T_{r}\right)$ higher than the auto-ignition temperature of the mixture $\left(T_{i g n}\right)$ while maintaining the temperature rise across the flame $(\Delta T)$ lower than the auto-ignition temperature [11]. The autoignition temperature of a mixture is calculated using a well-stirred reactor (WSR) model with a residence time of $1 \mathrm{~s}$. Moreover, as noted by Desai et al. [18], by increasing the inlet mixture reactivity (i.e. preheating it to the auto-ignition temperature), the laminar flame speed is no longer an intrinsic property of the mixture only, but depends on the domain size. For that reason, a domain sensitivity analysis is performed to identify $T_{r}$ for which the solution is domain-independent.

Table 1 lists the thermochemical conditions of the cases under study. For a physically relevant comparison, a lean ammonia MILD flame (MILD1) is compared with two conventional cases which were selected by matching either the flame temperature (A1) or the laminar flame speed (B1).

Table 1 Inlet thermochemical conditions of considered cases. All conditions are at pressure $P=1$ atm.

\begin{tabular}{ccrrrrrrr}
\hline Case & $\phi$ & $X_{\mathrm{NH}_{3}}$ & $X_{\mathrm{O}_{2}}$ & $X_{\mathrm{N}_{2}}$ & $T_{r}[\mathrm{~K}]$ & $T_{p}[\mathrm{~K}]$ & $S_{L}[\mathrm{~cm} / \mathrm{s}]$ & $\delta_{t h}[\mathrm{~mm}]$ \\
\hline MILD1 & 0.7 & 0.07 & 0.08 & 0.85 & 1200 & 1797 & 33.0 & 2.15 \\
A1 & 0.7 & 0.13 & 0.14 & 0.73 & 700 & 1797 & 11.0 & 2.70 \\
B1 & 0.7 & 0.16 & 0.18 & 0.66 & 750 & 2047 & 33.0 & 1.17 \\
\hline
\end{tabular}

To investigate the effects of turbulence on the flames, two-dimensional high-fidelity direct numerical simulations are performed for the cases MILD1, and B1, whose conditions are described in Table 1. Simulations of propagating flames in a channel are conducted by using the in-house KARFS code (KAUST Adaptive Reacting Flow Solver). Readers may refer to Hernández Pérez et al. [19] and Desai et al. [20] for more details regarding the code performance and capabilities. The compressible Navier-Stokes, continuity, species, and energy equations are solved by using an eighth-order central finite-difference operator for spatial discretization while a six-stage fourth-order explicit Runge-Kutta scheme is used for the temporal integration of the solution. To eliminate spurious high-frequency fluctuations, a tenth-order filter is applied. Periodic boundary conditions are applied in the vertical axis while Navier-Stokes characteristic boundary conditions are used for the outflow boundary [21, 22]. The solution from the one-dimensional steady-state freely propagating flame configurations are mapped onto the two-dimensional domain as an initial condition and turbulent fluctuations are imposed. An isotropic turbulent field was initialized by prescribing the energy spectrum in spectral space [23]. Linear turbulent forcing, as described in Ref. [24], is applied in physical space within certain computational domain range to maintain the original level of turbulence in the fresh mixture that lies below a temperature cutoff value (which is specific for each case).

The associated turbulence, non-dimensional and domain discretization parameters are listed in Table 2. To investigate fundamental turbulent burning characteristics, the ratios of turbulent intensity to the laminar flame speed $\left(u^{\prime} / S_{L}=10\right)$, and integral length scale to the laminar thermal thickness $\left(l_{T} / \delta_{t h}=2\right)$ are identical in each case. Both turbulent flames 
fall into the thin reaction zone regime according to the Borghi regime diagram [25]. The turbulent Reynolds number is defined as $R e=\frac{u^{\prime} l_{I}}{v_{0}}$, the Karlovitz number is defined as $K a=\left(\frac{\delta_{t h}}{\eta_{k}}\right)^{2}$ and the Damkhöler number is defined as $D a=\frac{l_{l} \cdot \delta_{t h}}{s_{L} \cdot u^{\prime}}$, where $v_{0}$ is the unburned gas kinematic viscosity, $l_{l}$ is the integral length scale and $\eta_{k}$ is the Kolmogorov length scale. The computational domain in each case is discretized using a uniform grid consisting of 120,000 points. The grid spacing was set to adequately resolve all the relevant spatial scales such as Kolmogorov length scale $\left(\eta_{k}\right)$ and reaction zone with at least 20 grid points.

Table 2 Turbulence parameters for two-dimensional DNS setup.

\begin{tabular}{crrrrrrr}
\hline Case & $u^{\prime}[\mathrm{m} / \mathrm{s}]$ & $l_{T}[\mathrm{~mm}]$ & $\eta_{k}[\mathrm{~mm}]$ & $\Delta x[\mathrm{~mm}]$ & $\mathrm{Re}$ & $\mathrm{Da}$ & $\mathrm{Ka}$ \\
\hline MILD1 & 3.4 & 4.3 & 0.15 & 0.1 & 86 & 0.2 & 46 \\
B1 & 3.3 & 2.3 & 0.07 & 0.05 & 99 & 0.2 & 50 \\
\hline
\end{tabular}

\section{Results and Discussion}

To analyze the effects of preheating and dilution of lean reactant mixtures on the heat release rate (HRR), the profiles corresponding to cases MILD1, A1 and B1 are shown in Fig. 11.a). Note that the results are plotted versus the temperature-based progress variable, defined as $c_{T}=\frac{T-T_{r}}{T_{p}-T_{r}}$, where $T_{r}$ and $T_{p}$ denote reactant and product gas temperature, respectively. The peak of HRR in all cases is found at the downstream region. For case MILD1, the HRR decreases as compared with the flame which has the same laminar flame speed (B1), and it is similar to that of flame A1 (which has the same product temperature). Comparing the HRR profile of MILD1 and A1, it spreads more for the MILD1 case due to the highly reactive nature of the inlet mixture - a significant level of HRR is observed in the upstream reactant zone.

(a)

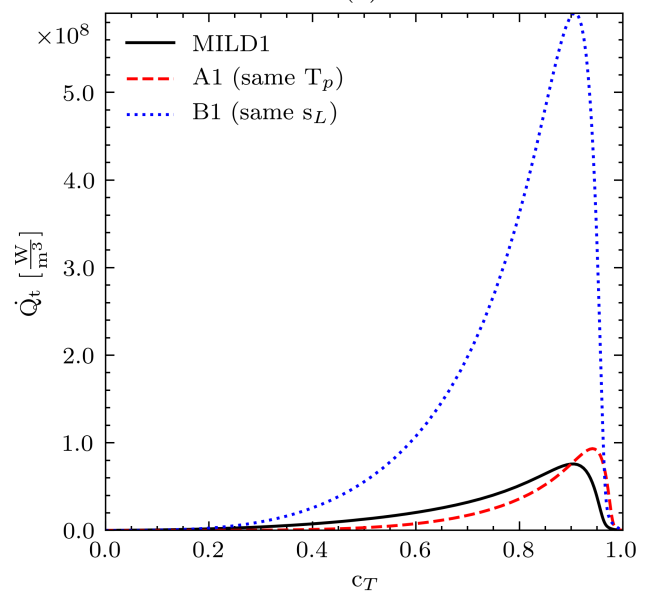

(b)

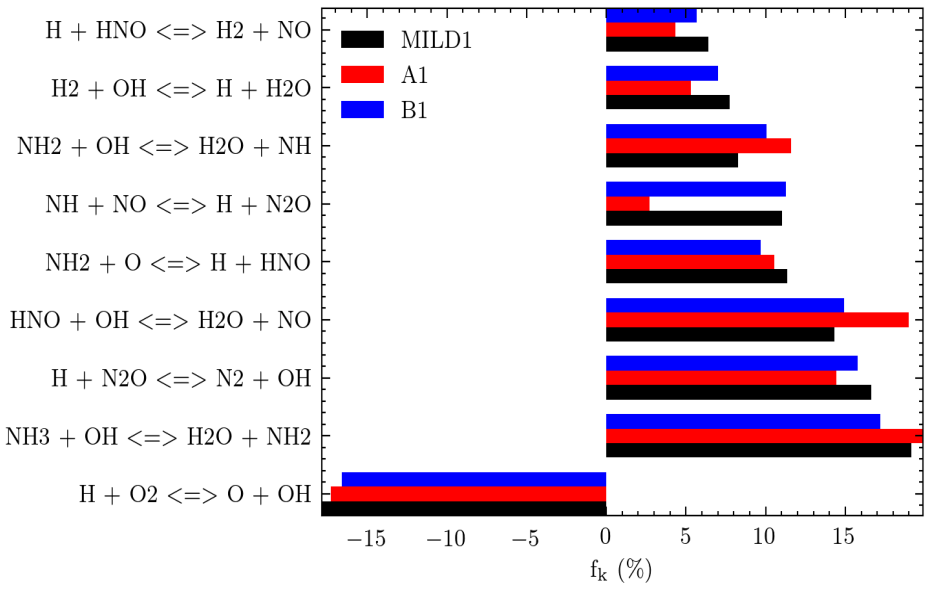

Fig. 1 Comparison of (a) heat release profiles in progress variable space, (b) fractional contribution from dominant HRR reactions for one-dimensional laminar flames.

Quantitative analysis on dominant HRR reactions is investigated by calculating the volumetric heat release rates on a per-reaction basis as follows:

$$
h_{k}=\omega_{k} \sum_{\alpha} h_{f, \alpha}^{0}\left(v_{k, \alpha}^{\prime \prime}-v_{k, \alpha}^{\prime}\right) \quad(k=1, \ldots, K) \quad\left(\frac{\mathrm{W}}{\mathrm{m}^{3}}\right),
$$

where $\omega_{k}$ is the net rate of progress of reaction $k$ (also known as net reaction rate), $h_{f, \alpha}^{0}$ is enthalpy of formation of species $\alpha, v_{k, \alpha}^{\prime \prime}$ and $v_{k, \alpha}^{\prime}$ are stoichiometric coefficients of species $\alpha$ in reaction $k$. The total volumetric HRR is then 
obtained by summing $h_{k}$ over all reaction steps

$$
h_{T}=\sum_{k} h_{k}\left(\frac{\mathrm{W}}{\mathrm{m}^{3}}\right) .
$$

The volume-integrated HRR from each reaction $k$ and total HRR are calculated as

$$
\begin{aligned}
& H_{k}=\int_{L} h_{k} \mathrm{dx} \quad\left(\frac{\mathrm{W}}{\mathrm{m}^{2}}\right), \\
& H_{T}=\int_{L} h_{T} \mathrm{dx} \quad\left(\frac{\mathrm{W}}{\mathrm{m}^{2}}\right),
\end{aligned}
$$

where $L$ denotes the computational domain. Consequently, the fractional contribution of reaction $k$ on the total HRR is defined as

$$
f_{k}=\frac{H_{k}}{H_{T}} \times 100
$$

Following the fractional contribution analysis explained above, the dominant HRR reactions are identified for each case. As shown in Figure 1 (b), the chain-branching reaction $\mathrm{H}+\mathrm{O}_{2} \rightleftharpoons \mathrm{O}+\mathrm{OH}$ is the main endothermic reaction with a fractional contribution to the total heat release rate of more than $15 \%$ for each case. The reaction $\mathrm{NH}_{3}+\mathrm{OH} \rightleftharpoons \mathrm{H}_{2} \mathrm{O}+\mathrm{NH}_{2}$ is a major exothermic reaction imparting more than $17 \%$ fractional contribution to the total HRR for the flames MILD1, A1 and B1. Preheating and dilution of the reactant mixture promotes heat release from reactions $\mathrm{H}_{2}+\mathrm{OH} \rightleftharpoons \mathrm{H}+\mathrm{H}_{2} \mathrm{O}$ and $\mathrm{H}+\mathrm{N}_{2} \mathrm{O} \rightleftharpoons \mathrm{N}_{2}+\mathrm{OH}$, as compared with the corresponding normal flames. The increased reactivity and reduced oxygen content of the inlet mixture promote hydrogen related reactions, which are enhanced by diffusion of hydrogen. Note that fractional contribution of one of the preponderant reactions $\mathrm{NH}_{2}+\mathrm{OH} \rightleftharpoons \mathrm{H}_{2} \mathrm{O}+\mathrm{NH}$ is reduced to approximately $7 \%$ for the case MILD1.

With the understanding of HRR dominant chemical pathways, the NO emission is subsequently analyzed. For the same flame speed, a lower NO emission is observed, which is inferred by comparing the NO mole fraction between MILD1 and A1 in Figure 2(a). Although the NO levels in A1 and MILD1 are almost the same, the flame speed of flame $\mathrm{A} 1$ is 3 times slower than that of MILD1. As reported in the literature, $\mathrm{NO}_{\mathrm{x}}$ are mainly produced from fuel-NO pathway via HNO intermediate species for all equivalence ratios [1, 5]. Moreover, as pointed out by Miller et al. [26], under certain range of oxygen amount and temperature, the thermal- $\mathrm{DeNO}_{\mathrm{x}}$ process can enhance the $\mathrm{NO}$ reduction by promoting reaction $\mathrm{NH}_{2}+\mathrm{NO}$. Figure 2(b) illustrates the $\mathrm{NO}$ production rate from $\mathrm{HNO}+\mathrm{OH} \rightleftharpoons \mathrm{H}_{2} \mathrm{O}+\mathrm{NO}$ (blue color), the dominant fuel-NO production pathway, and $\mathrm{NO}$ consumption rate from $\mathrm{NH}_{2}+\mathrm{NO} \rightleftharpoons \mathrm{H}_{2} \mathrm{O}+\mathrm{N}_{2}$ (red color), the key reaction in the thermal-DeNO $\mathrm{x}_{\mathrm{x}}$ process, overlaid with total $\mathrm{NO}$ (black color) production profiles. Note that the profiles are shown for MILD1 flame (solid line) and A1 flame (dashed line), which have nearly the same emission levels. By looking at the total NO production rate, it is noticed that the NO is consumed and produced early in the reaction zone for the flame MILD1. Furthermore, the consumption by thermal-DeNO $\mathrm{x}_{\mathrm{x}}$ process is dominant for low and intermediate temperature range in MILD1.

The influence of turbulence on flames MILD1 and B1 (which have same laminar flame speed) is discussed next. Figure 3 illustrates two-dimensional instantaneous snapshots of the temperature field at a time instant of $10 \times \tau_{\text {eddy }}$ where $\tau_{e d d y}$ is the eddy turnover time defined as the ratio $l_{\mathrm{T}} / u^{\prime}$ with $l_{\mathrm{T}}$ and $u^{\prime}$ being the integral length scale and turbulent intensity, respectively. Due to the high dilution of MILD1 flame, its temperature rise is lower and its flame thickness is larger. On the other hand, the level of wrinkling in B1 flame is higher. The instantaneous spatial distribution of heat release rate is also shown in Figure 4 The higher heat release in positive curvature (convex towards reactants) is observed for both cases, while positive heat release values are also noted in concave regions for the MILD1 flame. 

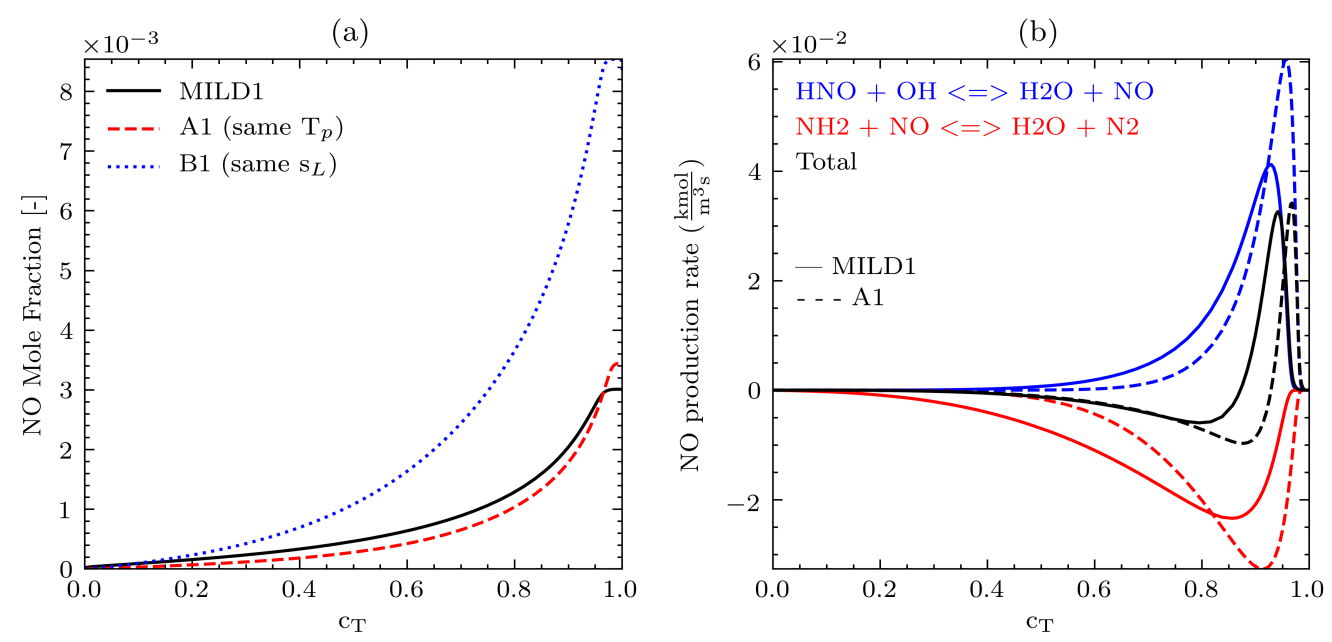

Fig. 2 Comparison of NO (a) mole fraction (b) production rate in progress variable space for one-dimensional laminar flames. The solid line corresponds to MILD1, while the dashed line corresponds to A1.

(a)

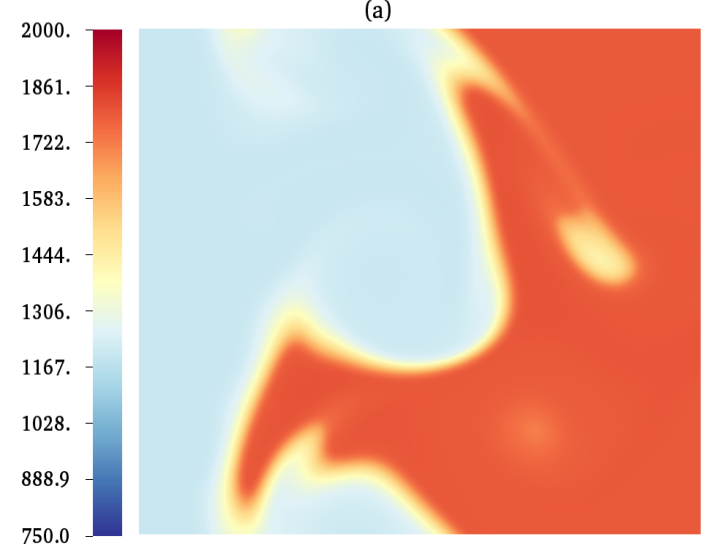

(b)

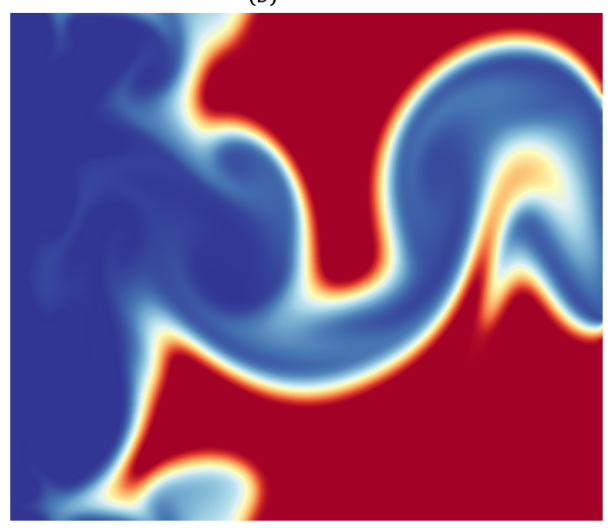

Fig. 3 Temperature distribution at time $\tau_{\text {eddy }}=10$ for the cases (a) MILD1 and (b) B1.
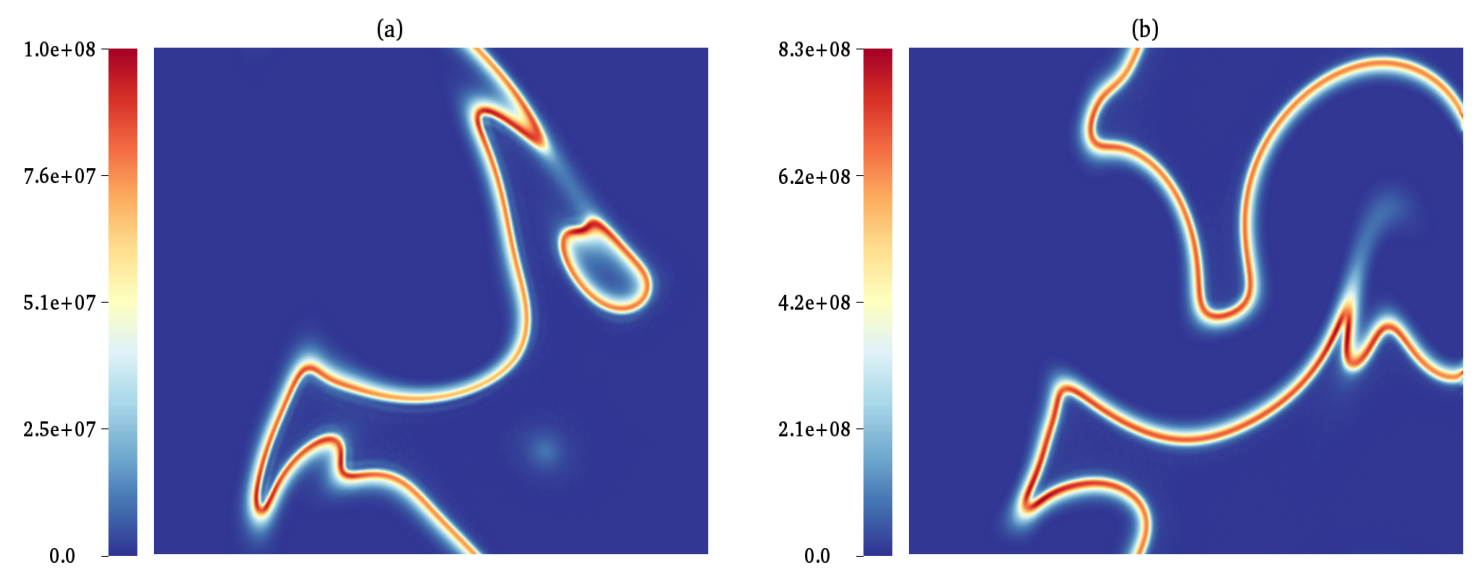

Fig. 4 Heat release rate distribution at time $\tau_{\text {eddy }}=10$ for the cases (a) MILD1 and (b) B1.

Figure 5 shows scatter plots of the normalized heat release versus temperature-based progress variable for cases MILD1 and B1. Heat release is normalized by the corresponding maximum value of the rate of heat release from 
a one-dimensional laminar flame. In each case, the associated laminar heat release profile (displayed in solid black line) is included. A wider range of scattering is observed in the preheat zone $\left(0.3<c_{T}<0.7\right)$ for case B1, while for case MILD1 it is mainly observed in the reaction zone $\left(0.7<c_{T}<0.8\right)$. This implies a broader reaction zone for the ammonia MILD flame.

(a)

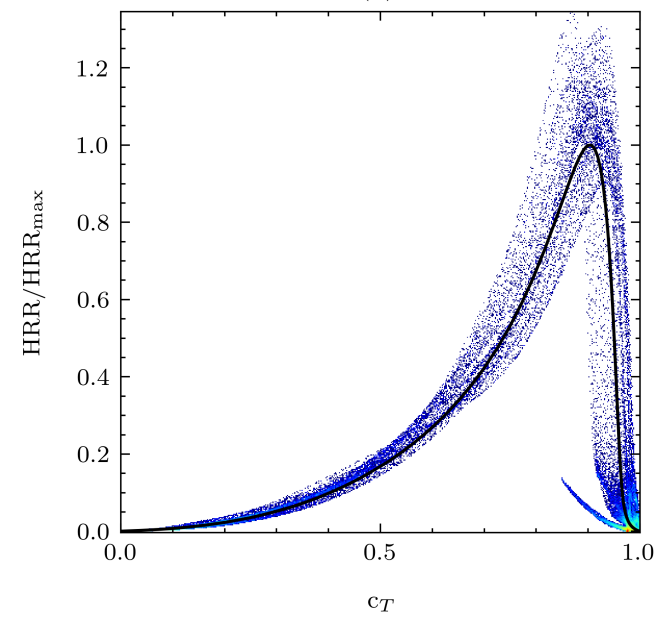

(b)

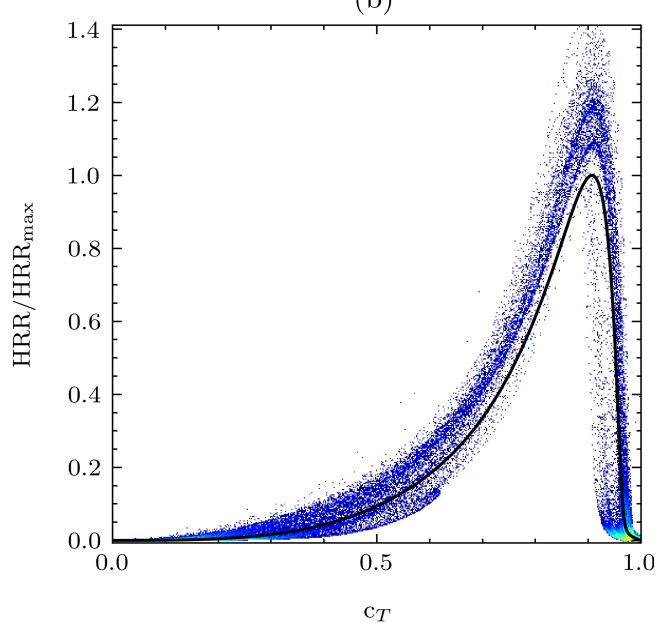

Fig. 5 HRR profile at the time $\tau_{\text {eddy }}=10$ for the case (a) MILD1 and (b) B1.

The global burning velocity characteristics are examined based on the fuel consumption speed as well as the flame surface area. The former is given by [27]

$$
S_{T}=\frac{1}{\rho_{u} Y_{u, F} A_{0}} \int_{V} \dot{\omega}_{F} \mathrm{dV},
$$

where $\rho, Y_{u, F}, \dot{\omega}_{F}$, and $A_{0}$ stand for density of fresh mixture, mass fraction of fuel in the fresh mixture, reaction rate of fuel, and projected flame area onto the direction of the flame propagation, respectively. Figure 6 displays the ratio between normalized flame speed and normalized flame surface i.e stretch factor of each flames. Note that division by normalized flame surface eliminates the effect of surface area increase. The flame surface is based on the isolevel of progress variable $c_{T}=0.87$, where the peak of heat release rate occurs. The turbulent flame speed increase for case B1 is slightly higher when contrasted to that for case MILD1. Nevertheless, the flame enhancement is mainly due to area increase, which verifies Damköhler's first hypothesis for the MILD and normal flames considered in this study [28], which is also shown in Figure 7

\section{Conclusions}

A computational study of lean ammonia premixed flame characteristics in MILD and normal combustion was conducted for one-dimensional laminar and two-dimensional turbulent flame configurations. The one-dimensional results revealed that the heat release profile is attenuated for the MILD flame as compared to the normal flame that has identical laminar flame speed. Moreover, dominant reaction pathway analysis indicated that in the MILD flame the contribution of hydrogen-related reactions is promoted. Analysis of turbulent flame speed and surface area indicates that the flame speed and flame surface area are well correlated for both the MILD and non-MILD flames, with the flame speed enhancement being slightly larger for the non-MILD flame.

\section{Acknowledgments}

This work was sponsored by King Abdullah University of Science and Technology (KAUST). Computational resources were provided by the KAUST Supercomputing Laboratory (KSL). 


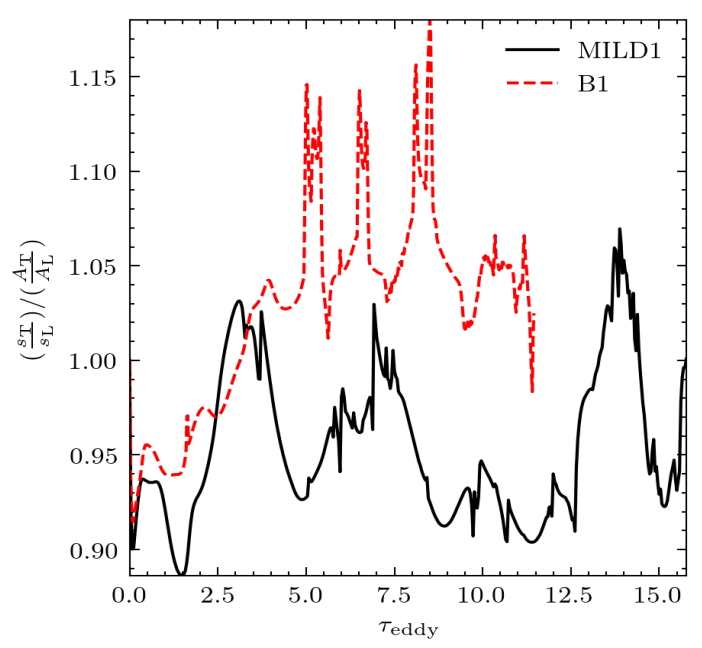

Fig. 6 Temporal evolution of stretch factor.

(a)

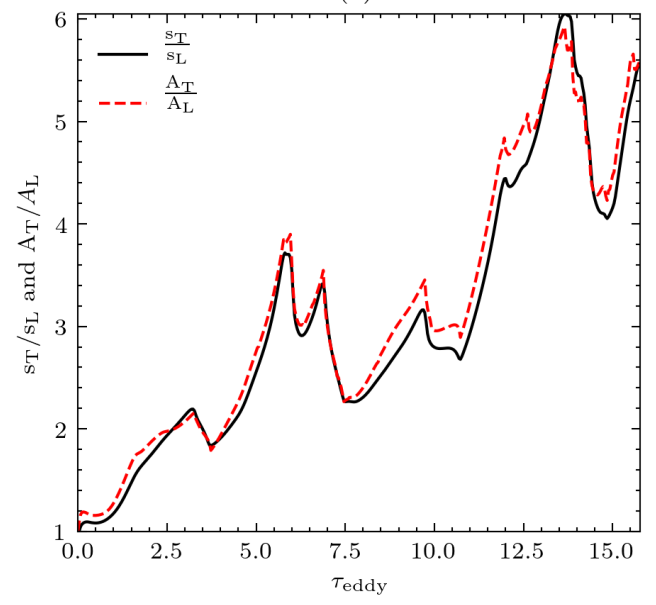

(b)

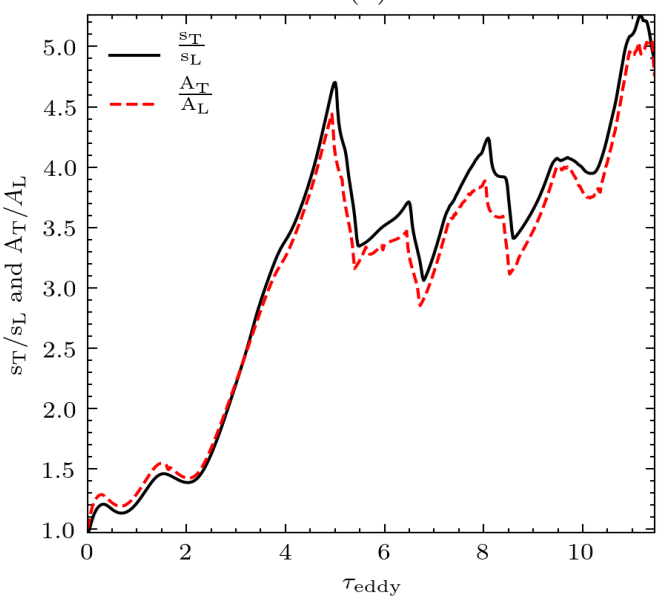

Fig. 7 Temporal evolution of normalized flame speed and normalized flame surface area for cases (a) MILD1 (b) B1.

\section{References}

[1] Kobayashi, H., Hayakawa, A., Somarathne, K. K. A., and Okafor, E. C., "Science and technology of ammonia combustion," Proceedings of the Combustion Institute, Vol. 37, No. 1, 2019, pp. 109-133.

[2] Koike, M., Miyagawa, H., Suzuoki, T., and Ogasawara, K., "Ammonia as a hydrogen energy carrier and its application to internal combustion engines," Journal of the Combustion Society of Japan, Vol. 58, No. 184, 2016, pp. $99-106$.

[3] Ezzat, M., and Dincer, I., "Development and assessment of a new hybrid vehicle with ammonia and hydrogen," Applied Energy, Vol. 219, 2018, pp. 226-239.

[4] Mørch, C. S., Bjerre, A., Gøttrup, M. P., Sorenson, S. C., and Schramm, J., "Ammonia/hydrogen mixtures in an SI-engine: Engine performance and analysis of a proposed fuel system," Fuel, Vol. 90, No. 2, 2011, pp. 854-864.

[5] Valera-Medina, A., Xiao, H., Owen-Jones, M., David, W., and Bowen, P., “Ammonia for power," Progress in Energy and Combustion Science, Vol. 69, 2018, pp. 63-102.

[6] Dimitriou, P., and Javaid, R., "A review of ammonia as a compression ignition engine fuel," International Journal of Hydrogen Energy, Vol. 45, No. 11, 2020, pp. 7098-7118.

[7] Hayakawa, A., Arakawa, Y., Mimoto, R., Somarathne, K. K. A., Kudo, T., and Kobayashi, H., "Experimental investigation of stabilization and emission characteristics of ammonia/air premixed flames in a swirl combustor," International Journal of Hydrogen Energy, Vol. 42, No. 19, 2017, pp. 14010-14018. 
[8] Kurata, O., Iki, N., Matsunuma, T., Inoue, T., Tsujimura, T., Furutani, H., Kobayashi, H., and Hayakawa, A., "Performances and emission characteristics of NH3-air and NH3CH4-air combustion gas-turbine power generations," Proceedings of the Combustion Institute, Vol. 36, No. 3, 2017, pp. 3351-3359.

[9] Hayakawa, A., Goto, T., Mimoto, R., Arakawa, Y., Kudo, T., and Kobayashi, H., "Laminar burning velocity and Markstein length of ammonia/air premixed flames at various pressures," Fuel, Vol. 159, 2015, pp. 98-106.

[10] Wünning, J. G., "Flameless Oxidation,” 6th HiTACG Symposium, , No. October, 2005, p. 13.

[11] Cavaliere, A., and de Joannon, M., "Mild combustion," Progress in Energy and Combustion science, Vol. 30, No. 4, 2004 , pp. 329-366.

[12] Swaminathan, N., "Physical insights on mild combustion from dns," Frontiers in Mechanical Engineering, Vol. 5, 2019 , p. 59.

[13] Doan, N. A. K., and Swaminathan, N., "Role of radicals on MILD combustion inception," Proceedings of the Combustion Institute, Vol. 37, No. 4, 2019, pp. 4539-4546.

[14] Sorrentino, G., Sabia, P., Bozza, P., Ragucci, R., and de Joannon, M., "Low-NOx conversion of pure ammonia in a cyclonic burner under locally diluted and preheated conditions," Applied Energy, Vol. 254, 2019, p. 113676.

[15] Ariemma, G., Sabia, P., Sorrentino, G., Bozza, P., De Joannon, M., and Ragucci, R., "Influence of water addition on MILD ammonia combustion performances and emissions," Proceedings of the Combustion Institute, 2020.

[16] Goodwin, D., "Cantera: Object-oriented software for reacting flows," California Institute for Technology (Caltech). http://www. cantera. org (accessed July, 2007), 2005.

[17] Otomo, J., Koshi, M., Mitsumori, T., Iwasaki, H., and Yamada, K., "Chemical kinetic modeling of ammonia oxidation with improved reaction mechanism for ammonia/air and ammonia/hydrogen/air combustion," International Journal of Hydrogen Energy, Vol. 43, No. 5, 2018, pp. 3004-3014.

[18] Desai, S., Sankaran, R., and Im, H. G., "Unsteady deflagration speed of an auto-ignitive dimethyl-ether DME/air mixture at stratified conditions," Proceedings of the Combustion Institute, Vol. 37, No. 4, 2019, pp. 4717-4727.

[19] Hernández Pérez, F. E., Mukhadiyev, N., Xu, X., Sow, A., Lee, B. J., Sankaran, R., and Im, H. G., "Direct numerical simulations of reacting flows with detailed chemistry using many-core/GPU acceleration," Comput. Fluids, Vol. 173, 2018.

[20] Desai, S., Kim, Y. J., Song, W., Luong, M. B., Pérez, F. E. H., Sankaran, R., and Im, H. G., "Direct numerical simulations of turbulent reacting flows with shock waves and stiff chemistry using many-core/GPU acceleration," Computers \& Fluids, 2020, p. 104787.

[21] Yoo, C. S., Wang, Y., Trouvé, A., and Im, H. G., "Characteristic boundary conditions for direct simulations of turbulent counterflow flames," Combust. Theor. Model., Vol. 9, No. 4, 2005, pp. 617-646.

[22] Yoo, C. S., and Im, H. G., "Characteristic boundary conditions for simulations of compressible reacting flows with multidimensional, viscous and reaction effects," Combust. Theor. Model., Vol. 11, No. 2, 2007, pp. 259-286.

[23] Passot, T., and Pouquet, A., "Numerical simulation of compressible homogeneous flows in the turbulent regime," J. Fluid Mech., Vol. 181, 1987, pp. 441-466.

[24] Bassenne, M., Urzay, J., Park, G. I., and Moin, P., "Constant-energetics physical-space forcing methods for improved convergence to homogeneous-isotropic turbulence with application to particle-laden flows," Phys. Fluids, Vol. 28, 2016.

[25] Peters, N., Turbulent combustion, Cambridge university press, 2000.

[26] Miller, J. A., Branch, M., and Kee, R. J., "A chemical kinetic model for the selective reduction of nitric oxide by ammonia," Combustion and Flame, Vol. 43, 1981, pp. 81-98.

[27] Poinsot, T., and Veynante, D., Theoretical and numerical combustion, RT Edwards, Inc., 2005.

[28] Law, C. K., Combustion physics, Cambridge university press, 2006. 